\title{
The object of the exercise...
}

Breathlessness, wheeze, chest tightness and cough can be experienced at rest or during or after physical exercise by individuals of all ages and lifestyles, from the sedentary to the elite athlete. When associated with physical exercise, these symptoms may be suggestive of asthma, as well as other cardiopulmonary diseases. Although exercise is a common asthma trigger, exercise-associated symptoms are neither sensitive nor specific for exercise-induced bronchoconstriction (EIB) - $a \geq 10 \%$ decline in FEV 1 within 30 min of ceasing exercise - which is a cardinal manifestation of asthma. In addition to symptoms, variable airflow obstruction should be documented before a diagnosis of asthma is made. With the aim of improving the clinical understanding of the relationship between exercise and asthma, this issue of Breathe contains a series of review articles on this theme, with an emphasis on the potential pitfalls in diagnosis, stimulus and mechanisms of EIB and the challenges employed for its identification, together with the challenges of its management in elite athletes.

"All that wheezes is not asthma" is a well known aphorism. In addition to benign lung tumours and inhaled foreign bodies, laryngeal dysfunction should be considered as a cause in children, adolescents and young adults with exercise-induced respiratory symptoms. Previously labelled "vocal cord dysfunction", it is increasingly clear that exercise can induce different types of laryngeal obstruction. Although the diagnosis can be suspected on the clinical history of inspiratory wheeze and the observation of a flattened inspiratory flow-volume curve, the gold standard for diagnosis is the continuous laryngoscopy exercise test, now a routine investigation in some clinics.

Water loss from the lower respiratory tract is universally acknowledged as the stimulus for EIB. However, two theories, the thermal and the osmotic, have been proposed for the mecha nisms involved. Evidence for each is reviewed in this issue of Breathe. Increased vascular perme ability and leakage is common to both theories. The osmotic theory proposes that the rate of water loss is more important than the amount of water loss for the bronchoconstrictive effect.

The next two reviews look at the use of exercise challenge in the laboratory and the field for the identification of EIB. The importance of the rate as well as the amount of water loss is emphasised in the design of exercise challenge protocols. Optimal test conditions are described. However, exercise protocols need to be individualised, as subjects vary enormously in fitness level and age. It has recently been shown that reliable and valid data on cardiopulmonary exercise variables, such as maximal and peak $\mathrm{O}_{2}$ uptake and respiratory reserve, can be obtained from the exercise challenge test in the laboratory for identification of EIB.

Practical and safety issues with exercise chat lenge tests have led to the development of surrogate tests that utilise the osmotic hypothesis for the mechanism of EIB. In addition to the rationale for development, the protocols for and interpretation of the response to eucapnic voluntary hyperpnoea, as well as $4.5 \%$ hyperosmolar saline and mannitol tests, are presented and the appropriateness of each challenge for different clinical situations is discussed.

The final review focuses on the role of

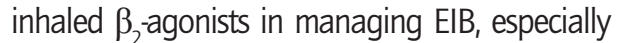
in elite athletes. In addition to beneficial effects, recent studies have highlighted adverse effects of these drugs, with the development of tolerance, resulting in a loss of bronchoprotection, reduction of bronchodilatation and a worsening of EIB. An algorithm for asthma management in the elite athlete is presented. Finally, past and present use of inhaled $\beta_{2}$-agonists in elite athletes is reviewed, together with the current status of regulations for use in competitive sports.

In conclusion, I would like to thank all the authors for their contributions and Breathe for the privilege of being guest editor for this series.
M. Sue-Chu

Dept of Lung Medicine, St Olav's Hospital, University Hospital of Trondheim, Institute of Circulation and Imaging, Norwegian University of Science and Technology, Trondheim, Norway

\section{Correspondence}

M. Sue-Chu Department of Lung Medicine

St. Olavs Hospital

Postbox 3250 Sluppen

7006 Trondheim

Norway

msuechu@online.no

\section{Provenance}

Commissioned introduction, not peer reviewed.

Competing interests

None declared. 